\title{
GRB 130427A: A Nearby Ordinary Monster
}

\author{
A. Maselli, ${ }^{1 *}$ A. Melandri, ${ }^{2}$ L. Nava ${ }^{2,3}$ C. G. Mundell, ${ }^{4}$ N. Kawai, ${ }^{5,6}$ S. Campana, ${ }^{2}$ S. Covino, ${ }^{2}$ \\ J. R. Cummings, ${ }^{7}$ G. Cusumano, ${ }^{1}$ P. A. Evans, ${ }^{8}$ G. Ghirlanda, ${ }^{2}$ G. Ghisellini, ${ }^{2}$ C. Guidorzi, ${ }^{9}$ \\ S. Kobayashi, ${ }^{4}$ P. Kuin, ${ }^{10}$ V. La Parola, ${ }^{1}$ V. Mangano, ${ }^{1,11}$ S. Oates, ${ }^{10}$ T. Sakamoto, ${ }^{12}$ M. Serino, ${ }^{6}$ \\ F. Virgili, ${ }^{4}$ B.-B. Zhang, ${ }^{11}$ S. Barthelmy, ${ }^{13}$ A. Beardmore, ${ }^{8}$ M. G. Bernardini, ${ }^{2}$ D. Bersier, ${ }^{4}$ \\ D. Burrows, ${ }^{11} \mathrm{G}$. Calderone, ${ }^{2,14} \mathrm{M}$. Capalbi, ${ }^{1}$ ]. Chiang, ${ }^{15} \mathrm{P}$. D'Avanzo, ${ }^{2}$ V. D'Elia, ${ }^{16,17}$ \\ M. De Pasquale, ${ }^{10}$ D. Fugazza, ${ }^{2}$ N. Gehrels, ${ }^{13}$ A. Gomboc, ${ }^{18,19}$ R. Harrison, ${ }^{4}$ H. Hanayama, ${ }^{20}$ \\ J. Japelj, ${ }^{18}$ J. Kennea, ${ }^{11}$ D. Kopac, ${ }^{18}$ C. Kouveliotou, ${ }^{21}$ D. Kuroda, ${ }^{22}$ A. Levan, ${ }^{23}$ D. Malesani, ${ }^{24}$ \\ F. Marshall, ${ }^{13}$ J. Nousek, ${ }^{11}$ P. $0^{\prime}$ Brien, $^{8}$ J. P. Osborne, ${ }^{8}$ C. Pagani ${ }^{8}$ K. L. Page, ${ }^{8}$ M. Page, ${ }^{10}$ \\ M. Perri, ${ }^{16,17}$ T. Pritchard, ${ }^{11}$ P. Romano o, ${ }^{1}$ Y. Saito, ${ }^{5}$ B. Sbarufatti, ${ }^{2,11}$ R. Salvaterra, ${ }^{25}{ }^{2}$ I. Steele, ${ }^{4}$ \\ N. Tanvir, ${ }^{8}$ G. Vianello, ${ }^{15}$ B. Wiegand, ${ }^{13}$ K. Wiersema, ${ }^{8}$ Y. Yatsu, ${ }^{5}$ T. Yoshii, ${ }^{5}$ G. Tagliaferri ${ }^{2}$
}

Long-duration gamma-ray bursts (GRBs) are an extremely rare outcome of the collapse of massive stars and are typically found in the distant universe. Because of its intrinsic luminosity $\left(L \sim 3 \times 10^{53}\right.$ ergs per second) and its relative proximity $(z=0.34)$, GRB 130427A reached the highest fluence observed in the $\gamma$-ray band. Here, we present a comprehensive multiwavelength view of GRB 130427A with Swift, the 2-meter Liverpool and Faulkes telescopes, and by other ground-based facilities, highlighting the evolution of the burst emission from the prompt to the afterglow phase. The properties of GRB 130427A are similar to those of the most luminous, high-redshift GRBs, suggesting that a common central engine is responsible for producing GRBs in both the contemporary and the early universe and over the full range of GRB isotropic energies.

$\mathrm{G}$ amma-ray burst (GRB) 130427A was the brightest burst detected with Swift (1), as well as with several $\gamma$-ray detectors onboard other space missions. It was also the brightest and longest burst detected above $100 \mathrm{MeV}$, with the most energetic photon detected at $95 \mathrm{GeV}$ (2). It was detected with Fermi-Gamma-ray Burst Monitor (GBM) (3) at $T_{0, \mathrm{GBM}}=47: 06.42$ UT on 27 April 2013. Hereafter, this time will be our reference time $T_{0}$. The Burst Alert Telescope (BAT) (4) onboard Swift triggered on GRB 130427A at time $(t)=$ $51.1 \mathrm{~s}$, when Swift completed a preplanned slew. The Swift slew to the source started at $t=148 \mathrm{~s}$ and ended at $t=192 \mathrm{~s}$. The Swift UltraViolet Optical Telescope (UVOT) (5) began observations at $t=181 \mathrm{~s}$, whereas observations with the Swift X-ray Telescope (XRT) (6) started at $t=195 \mathrm{~s}(7)$. The structure of the $\gamma$-ray light curve revealed by the Swift-BAT in the $15-$ to $350-\mathrm{keV}$ band (Fig. 1) can be divided in three main episodes: an initial peak, beginning at $t=0.1 \mathrm{~s}$ and peaking at $t=$ $0.5 \mathrm{~s}$; a second large peak, showing a complex structure with a duration of $\sim 20 \mathrm{~s}$; and a third, much weaker episode starting at $t \sim 120 \mathrm{~s}$, showing a fast rise/exponential decay behavior. The overall duration of the prompt emission was $T_{90}$ $(15$ to $150 \mathrm{keV})=276 \pm 5 \mathrm{~s}$ (the time containing $90 \%$ of the fluence) calculated over the first $1830 \mathrm{~s}$ of BAT observation from $T_{0}$, GBM. During the early phases of the $\gamma$-ray emission, strong spectral variability was observed (Fig. 1). A marked spectral hardening was observed during the prompt main event. With a total fluence $F=(4.985 \pm$ $0.002) \times 10^{-4} \mathrm{erg} \mathrm{cm}^{-2}$ in the 15 - to $150-\mathrm{keV}$ band, GRB 130427A reached the highest fluence ob- served for a GRB by Switt. The $0.02-$ to $10-\mathrm{MeV}$ fluence measured by Konus-Wind (8) for the main emission episode ( 0 to $18.7 \mathrm{~s})$ is $(2.68+/-0.01) \times$ $10^{-3} \mathrm{erg} \mathrm{cm}^{-2}$, with a spectrum peaking at $E_{\text {peak }}=$ $1028 \pm 8 \mathrm{keV}$, whereas the fluence of the emission episode at (120 to $250 \mathrm{~s}$ ) is $\sim 9 \times 10^{-5} \mathrm{erg} \mathrm{cm}^{-2}$, with a spectrum peaking at $\sim 240 \mathrm{keV}(9)$.

This event was extremely bright also in the optical and it was immediately detected with various robotic telescopes. In particular, the Raptor robotic telescope detected a bright optical counterpart already at $t=0.5 \mathrm{~s}(10)$. Optical spectroscopy of the afterglow determined the redshift to be $z=0.34$ (11); a UVOT UV grism spectrum (7) was also acquired. At this distance, the rest frame $1-\mathrm{keV}$ to $10-\mathrm{MeV}$ isotropic energy is $E_{\text {iso }}=$ $8.1 \times 10^{53} \mathrm{erg}$, and the peak luminosity is $L_{\text {iso }}=$ $2.7 \times 10^{53} \mathrm{erg} \mathrm{s}^{-1}$. According to the luminosity function of Salvaterra et al. (12), we expect one event like GRB 130427A every $>60$ years. In the nearby universe $(z \lesssim 0.4$, corresponding to an age of $\sim 10$ billion years), only a handful of long GRBs have been detected. These GRBs are usually characterized by a low overall isotropic energy $\left(E_{\text {iso }} \leq 10^{52} \mathrm{erg}\right)$ and are associated with supernovae (SNe) types Ib and Ic, characterized by broad spectral lines indicating high expansion velocities, called hypernovae (13). GRB 130427A is instead a powerful GRB, such as the ones typically detected at much higher redshifts $(z>1$, with a mean $z \sim 2$ corresponding to an age of $\sim 3$ billion years). The detection of a nearby and extremely powerful GRB gives us the opportunity to test, on the one hand, whether this GRB has the same properties of the cosmological GRBs and, on the other hand, whether also such bright GRBs are associated with SNe. Up to now, SNe have been associated only to under (or mildly) energetic GRBs in the local universe. Because a supernova associated with this burst, SN 2013cq, has been detected (14), we are now sure that $\mathrm{SNe}$ are also associated with very powerful GRBs, not only to low power bursts [fig. S6 and figure 1 of (14)]. Naive energetic arguments might suggest that in powerful GRBs, there is not enough power left for a strong SN; GRB 130427A definitively proves that this is not the case.

The overall behavior of the $\mathrm{x}$-ray afterglow light curve has been characterized with the main contribution of the XRT onboard Swift and two additional relevant detections from the Monitor of All-sky X-ray Image (MAXI) experiment (15) in the gap between the first and the second SwiftXRT observations (Fig. 2). The early light curve, starting from $t=260 \mathrm{~s}$, is characterized by an initially steep decay with a slope $\alpha_{0, \mathrm{x}}=3.32 \pm$ 0.17 , which is consistent with high-latitude emission $(16,17)$; a break at $t_{1, \mathrm{x}}=424 \pm 8 \mathrm{~s}$; and

${ }^{1}$ Istituto Nazionale di Astrofisica (INAF)-Istituto di Astrofisica Spaziale e Fisica Cosmica (IASF) Palermo, Via Ugo La Malfa 153 I-90146 Palermo, Italy. ${ }^{2}$ INAF-Osservatorio Astronomico di Brera, via E. Bianchi 46, I-23807 Merate, Italy. ${ }^{3}$ AstroParticule et Cosmologie, Université Paris Diderot, CNRS/IN2P3, Commissariat à l'Energie Atomique et aux Energies Alternatives/Institut de Recherches sur les lois Fondamentales de l'Univers, Observatoire de Paris, Sorbonne Paris Cité, France. ${ }^{4}$ Astrophysics Research Institute, Liverpool John Moores University, Liverpool Science Park, 146 Brownlow Hill, Liverpool L3 5RF, UK. 'Department of Physics, Tokyo Institute of Technology, 2-12-1 Ookayama, Meguro-ku, Tokyo 152-8551, Japan. ${ }^{6}$ Coordinated Space Observation and Experiment Research Group, RIKEN, 2-1 Hirosawa, Wako, Saitama 351-0198, Japan. ${ }^{7}$ University of Maryland, Baltimore County/ Center for Research and Exploration in Space Science \& Technology/ NASA Goddard Space Flight Center, Code 661, Greenbelt, MD 20771, USA. ${ }^{8}$ Department of Physics and Astronomy, University of Leicester, Leicester, LE1 7RH, UK. 'Department of Physics, University of Ferrara, via Saragat 1, I-44122, Ferrara, Italy. ${ }^{10}$ Mullard Space Science Laboratory, University College London, Holmbury St. Mary, Dorking, Surrey RH5 6NT, UK. ${ }^{11}$ Department of Astronomy and Astrophysics, Pennsylvania State University, 525 Davey Lab, University Park, PA 16802, USA. ${ }^{12}$ Department of Physics and Mathematics, Aoyama Gakuin University, 5-10-1 Fuchinobe, Chuo-ku, Sagamihara, Kanagawa 252-5258, Japan. ${ }^{13}$ NASA Goddard Space Flight Center, Greenbelt, MD 20771 USA. ${ }^{14}$ Dipartimento di Fisica "G. Occhialini," Università di MilanoBicocca, Piazza della Scienza 3, I-20126 Milano, Italy. ${ }^{15} \mathrm{~W}$. W. Hansen Experimental Physics Laboratory, Kavli Institute for Particle Astrophysics and Cosmology, Department of Physics, and SLAC National Accelerator Laboratory, Stanford University, Stanford, CA 94305, USA. ${ }^{16}$ INAF/Rome Astronomical Observatory, via Frascati 33, 00040 Monteporzio Catone (Roma), Italy. ${ }^{17}$ Agenzia Spaziale Italiana (ASI) Science Data Centre, Via Galileo Galilei, 00044 Frascati (Roma), Italy. ${ }^{18}$ Faculty of Mathematics and Physics, University of Ljubljana, Jadranska 19 1000, Ljubljana, Slovenia. ${ }^{19}$ Centre of Excellence Space-si, Askerceva cesta 12, 1000 Ljubljana, Slovenia. ${ }^{20}$ Ishigakijima Astronomical Observatory, National Astronomical Observatory of Japan, 1024-1 Arakawa, Ishigaki, Okinawa 907-0024, Japan. ${ }^{21}$ Space Science Office, VP62 NASA/Marshall Space Flight Center, Huntsville, AL 35812, USA ${ }^{22}$ Okayama Astrophysical Observatory, National Astronomical Observatory of Japan, 3037-5 Honjo, Kamogata, Asaguchi, Okayama 719-0232. ${ }^{23}$ Department of Physics, University of Warwick, Coventry CV4 7AL, UK. ${ }^{24}$ Dark Cosmology Centre (DARK), Niels Bohr Institute, University of Copenhagen, Juliane Maries Vej 30, 2100 Copenhagen, Denmark. ${ }^{25}$ INAF-IASF Milano, via E. Bassini 15, I-20133 Milano, Italy.

*Corresponding author. E-mail: maselli@ifc.inaf.it 


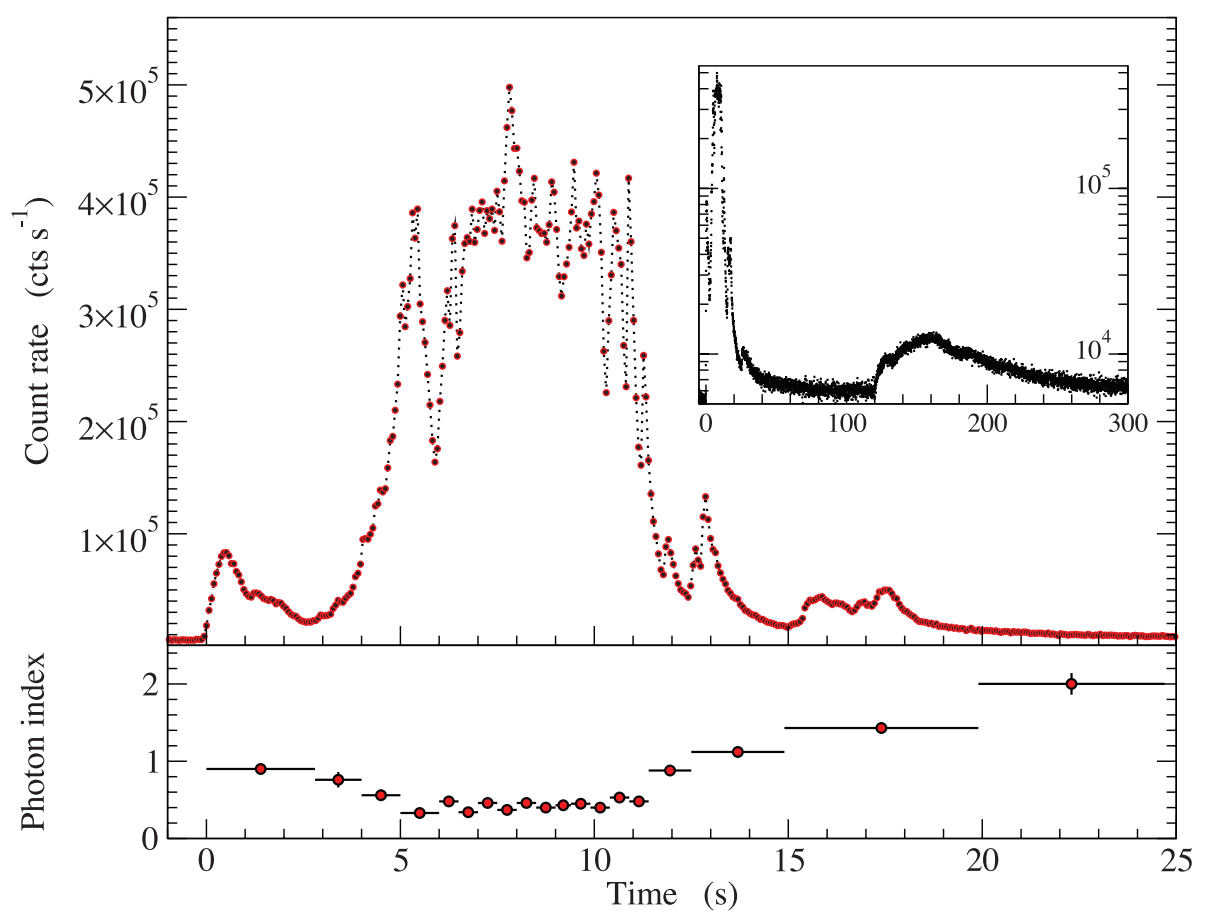

Fig. 1. Details of the Swift BAT light curve in the 15- to 350-keV band. (Top) The BAT light curve with a binning time of $64 \mathrm{~ms}$. (Inset) The BAT light curve up to $300 \mathrm{~s}$, plotted on a log intensity scale, showing a fast rise/exponential decay feature starting at $t \sim 120 \mathrm{~s}$. (Bottom) The photon index values of a power-law model fit to the BAT spectrum in the 15 to $150 \mathrm{keV}$ energy range.

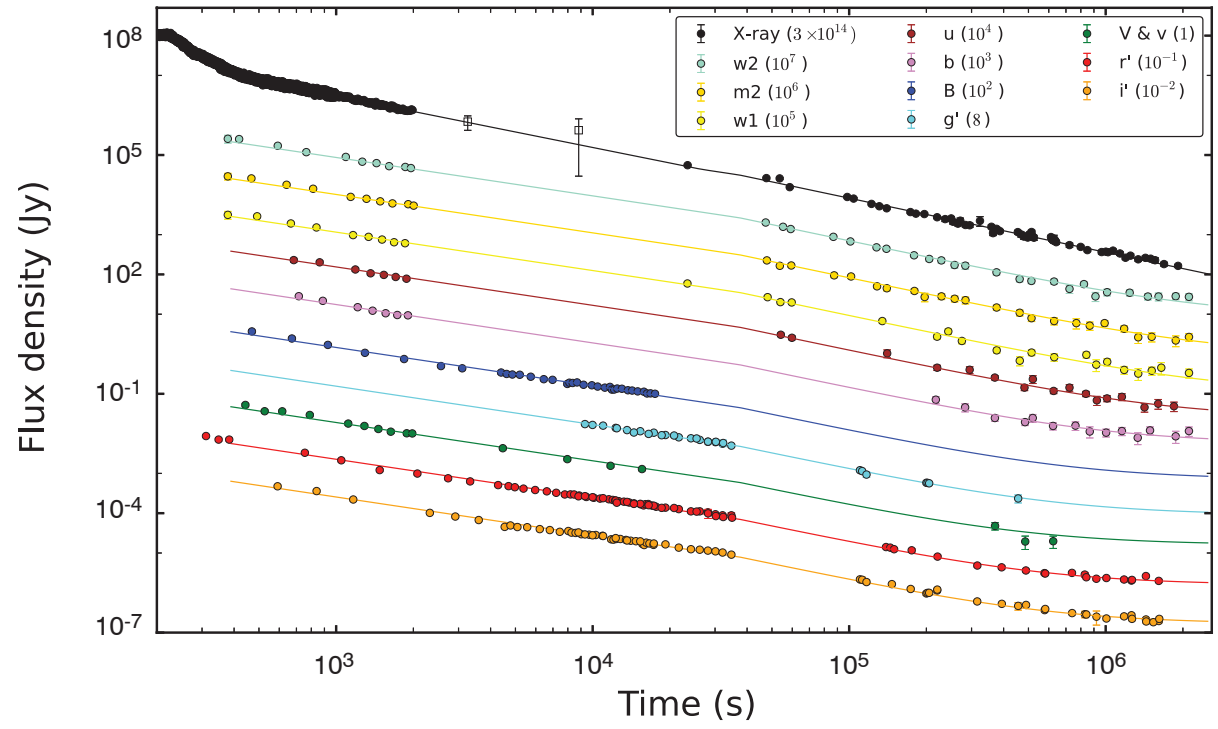

Fig. 2. Light curves for GRB 130427A in different wavebands. Swift UV and visible filters $(w 2, m 2$, $w 1, u, b, v) ; B, V, r^{\prime}$, and $i^{\prime}$ filters correspond to Faulkes Telescope North; $r^{\prime}$, and $i^{\prime}$ to the Liverpool Telescope; $g^{\prime}, r^{\prime}$, and $i^{\prime}$ to the MITSuME telescopes (7). The scaling factors for the flux density in different filters is shown in the inset; the scaling factor for the $x$-ray light curve (flux integrated in the 0.3 to $10 \mathrm{keV}$ range) (7) is also shown. The x-ray light curve also includes two MAXI data points at $t=3257 \mathrm{~s}$ and $t=8821 \mathrm{~s}$ (empty squares). The fit performed over all the light curves required 24 free parameters (curve normalizations, host galaxy optical flux in each band, three temporal slopes, and two breaks). Because of short-term low-level variability superposed to the long-term behavior and possibly residual inhomogeneity of optical data taken from different telescopes, we added in quadrature a $9 \%$ systematic error in the optical and $5 \%$ in the $x$-rays. Final fit yielded $\chi^{2}=543.02 / 565$ degrees of freedom. A contribution from the host galaxy has been taken into account in the optical bands by fitting a constant flux of $\sim 0.01$ millijansky for the reddest bands, corresponding to $r_{\mathrm{HG}}=21.26 \mathrm{mag}$ as tabulated in the Sloan Digital Sky Survey catalog. As a test of consistency, we performed the fit using a broken power law for the $B, g^{\prime}, r^{\prime}$, and $i^{\prime}$ filters individually. Uncertainties are larger, but we do find that the values of $t_{\text {break, }}$ as well as the decay indices before and after the break time, are still consistent, within the errors, with the values obtained by the overall fit. followed by a flatter decay with index $\alpha_{1, \mathrm{x}}=$ $1.28 \pm 0.01$. A further break at $t_{2, \mathrm{x}}=48 \pm 22 \mathrm{ks}$ is statistically needed (3.8 $)$ to account for a further steepening to $\alpha_{2, \mathrm{x}}=1.35 \pm 0.02$ (all errors are derived for $\Delta \chi^{2}=2.7$ )

The light curves in the optical and UV derived from the UVOT, as well as from groundbased telescopes (Liverpool telescope, Faulkes Telescope North, and MITSuME Telescopes) are shown in Fig. 2. All optical light curves are well fitted by a broken power law with $\alpha_{1}=$ $0.96 \pm 0.01, t_{\text {break }}=37.4_{-4.0}^{+4.7} \mathrm{ks}$, and $\alpha_{2}=$ $1.36_{-0.02}^{+0.01}$. Fitting the $\mathrm{x}$-ray light curve together with the optical ones, we find the same parameters from $26.6 \mathrm{ks}$ onward, but to fit the early part of the X-ray light curve, we need another powerlaw segment with a slope of $1.29_{-0.01}^{+0.02}$ and a break at $26.6_{-6.6}^{+4.5} \mathrm{ks}$ (Fig. 2). Therefore, from $26.6 \mathrm{ks}$ onward a common description of all the optical, UV, and x-ray behavior is possible, whereas at earlier times, an extra $\mathrm{x}$-ray component is required.

We interpret the early x-ray light curve (up to $26.6 \mathrm{ks}$ ) as the superposition of a standard afterglow (forward shock emission) and either the prolonged activity of the central engine or/and the contribution from the reverse shock emission (18-20). After $26.6 \mathrm{ks}$, the optical and x-ray light curves share the same behavior and decay slopes (Fig. 2), including a break at $t_{\text {break }} \sim 37 \mathrm{ks}$. This achromatic break is suggestive of a jet break, although the post-break decay $\left(\alpha_{2}=1.36\right)$ is shallower than predicted in the simplest theory [an increase in decay slope $>1$ would be expected (21)]. This could be due to additional components contributing to the flux, to a time dependence of the microphysical parameters governing the fraction of shock energy going to electrons $\left(\epsilon_{\mathrm{e}}\right)$ and magnetic field $\left(\epsilon_{\mathrm{B}}\right)$, or to the fact that we observed a canonical jet not exactly on axis, but still within the jet opening angle $(22,23)$.

Because the optical and the x-ray emission belong to the same spectral power-law segment, it is possible to constrain the characteristic frequencies of the afterglow spectra, in turn constraining the microphysical parameters of the relativistic shock. Additional information comes from the high-energy $\gamma$-ray emission (2). The $\gamma$-ray flux above $100 \mathrm{MeV}$ peaks at $\sim 20 \mathrm{~s}$. If this emission is due to afterglow radiation, the peak time implies a bulk Lorentz factor $\Gamma_{0} \sim 500(2,7)$. Furthermore, the presence of the giga-electon volt peak suggests a homogeneous circumburst density profile (24). Guided by these constraints in our choice of parameters, we used the BOXFIT code developed by van Eerten et al. (25) to model the afterglow. Rather than trying to perform a formal fit to the data, we checked whether this burst, with an unprecedented data coverage and richness, can be interpreted in the framework of the standard model for the afterglow emission, or else whether it forces us to abandon the standard framework. We gave more weight to the optical and higher energy fluxes because they carry 


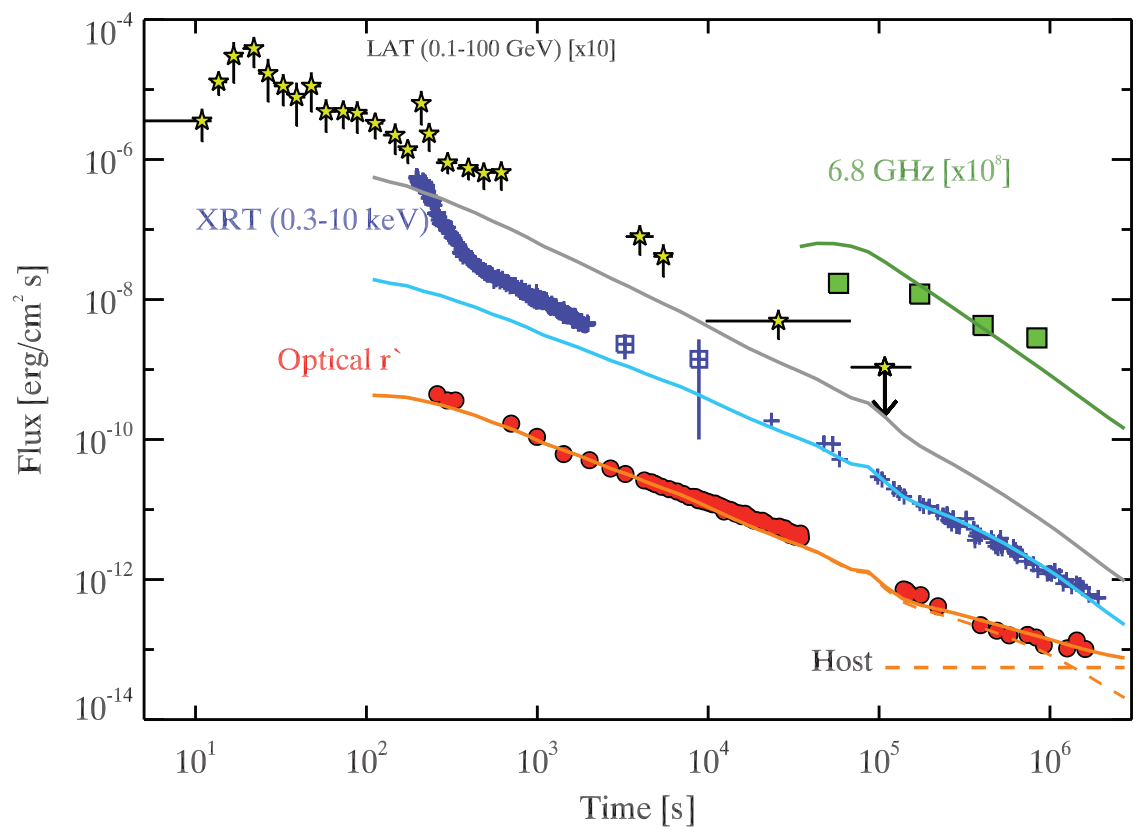

Fig. 3. Radio, optical, x-ray, and $\gamma$-ray light curves of GRB 130427A. Radio data are from (31): All measurements are taken at $6.8 \mathrm{GHz}$ except the later one, at $7.3 \mathrm{GHz}$. X-ray data are the same reported in Fig. 2 (7). LAT $\gamma$-ray data are from (2). Corresponding model predictions adopt a description in terms of the van Eerten et al. model (25). To properly fit the radio data, only a fraction of the electrons must be accelerated after $\sim 70$ ks (table S10) [discussion is available in (7)].

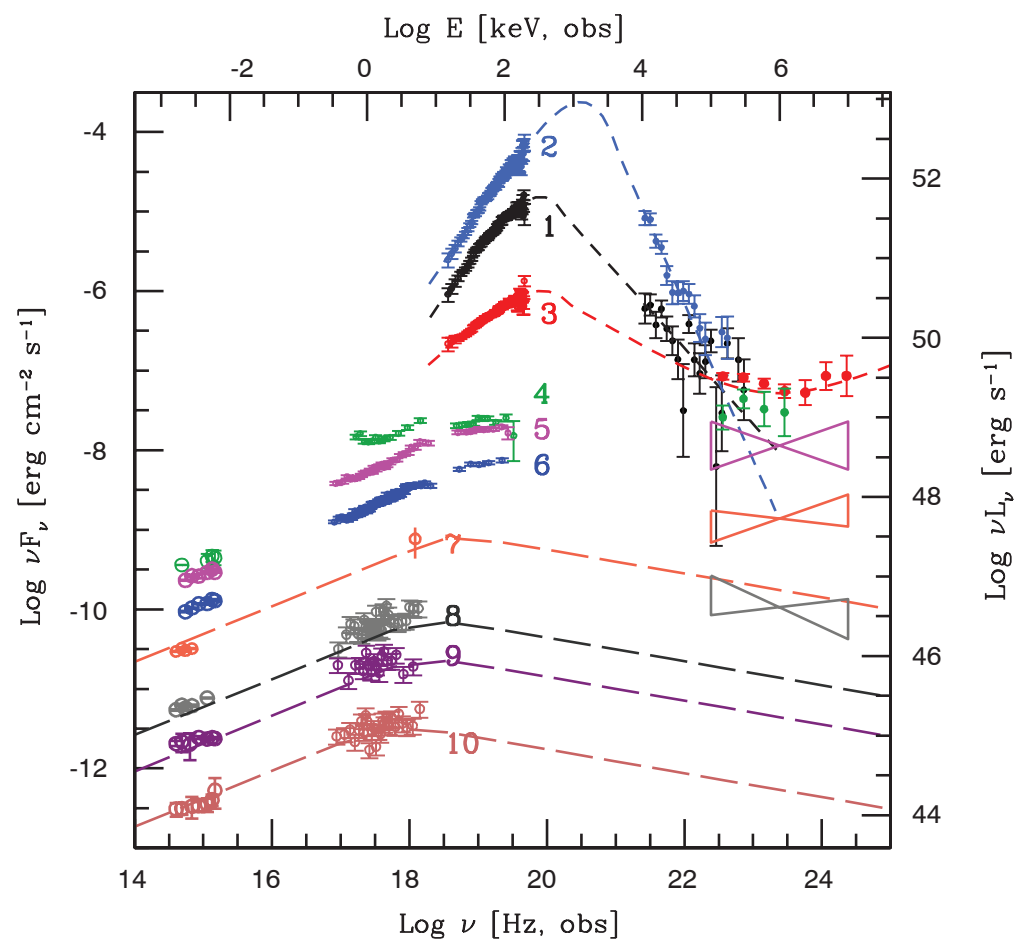

Fig. 4. Spectral Energy Distributions (SEDs) of GRB 130427A taken at different times, from the optical to the GeV bands. LAT data are from (2). For SED 1 and SED 2, the model is a Band function; for SED 3, the model is a Band + power law. Short dashed lines indicate phenomenological fits to the BAT + LAT data. Long dashed lines indicate results of the van Eerten et al. model (25), with parameters discussed in (7). The different SEDs refer to the following time intervals: 1,0 to $6.5 \mathrm{~s} ; 2,7.5$ to $8.5 \mathrm{~s} ; 3,8.5$ to $196 \mathrm{~s} ; 4,352$ to $403 \mathrm{~s} ; 5,406$ to $722 \mathrm{~s} ; 6,722-1830 \mathrm{~s} ; 7, \sim 3 \mathrm{ks} ; 8, \sim 23 \mathrm{ks} ; 9$, 59 ks; $10, \sim 220 \mathrm{ks}$ (host galaxy contribution has been subtracted in this case). From the optical SED analysis, the intrinsic extinction is negligible. most of the afterglow luminosity, which is orders of magnitude greater than the radio flux.

Neither reverse shock nor inverse Compton (IC) emission are included in the model, but this does not affect our conclusions, which primarily concern the late time synchrotron afterglow (7). The synchrotron flux predicted by the model reproduces the optical emission and the x-ray light curve after $\sim 10$ ks reasonably well, whereas the early x-ray flux is likely due to an additional component (Fig. 3). Our model underestimates the $\mathrm{GeV}$ emission, but this, given the large $\epsilon_{\mathrm{e}} / \epsilon_{\mathrm{B}}$ ratio, can be due to synchrotron Self-Compton emission, as envisaged by $(2,26,27)$. The model can also roughly reproduce the radio emission (7).

Consistent with the light curve analysis, the synchrotron flux predicted by the model reproduces reasonably well the optical and $\mathrm{x}$-ray parts of the spectral energy distribution (SED) of the afterglow (Fig. 4), but it underestimates the $\mathrm{GeV}$ emission. Although the model does not entirely reproduce the complexity shown by the data, it does capture the main features of the emission properties in the pure afterglow phase.

Overall, the properties of GRB 130427A are similar to those of the powerful GRBs typically seen at $z \sim 1-2$ [a comparison is available in (7)]. This is the most powerful GRB at $z<0.9$. It obeys the spectral energy correlations, such as the $E_{\text {peak }}-E_{\text {iso }}(28)$ correlation and the $E_{\text {peak }}-$ $L_{\text {peak }}(29)$ correlation. Interpreting as a jet break the break observed at $37 \mathrm{ks}$ makes GRB 130427A consistent with the collimation corrected energypeak energy correlation $(7,30)$. GRB 130427A is also associated with a supernova, extending the GRB-SN connection also to such powerful and high- $z$ bursts. GRB 130427A stands as an exceptional example indicating that a common engine is powering these huge explosions at all powers, and from the nearby to the very far, early universe.

References and Notes

1. A. Maselli et al., GRB Coordinates Network $\mathbf{1 4 4 4 8 ,}$ 1 (2013).

2. M. Ackermann et al., Science 343, 42-47 (2014).

3. C. Meegan et al., Astrophys. J. 702, 791-804 (2009).

4. S. D. Barthelmy et al., Space Sci. Rev. 120, 143-164 (2005).

5. P. W. A. Roming et al., Space Sci. Rev. 120, 95-142 (2005).

6. D. N. Burrows et al., Space Sci. Rev. 120, 165-195 (2005).

7. Materials and methods are available as supplementary materials on Science Online.

8. R. L. Aptekar et al., Space Sci. Rev. 71, 265-272 (1995).

9. S. Golenetskii et al., GRB Coordinates Network 14487, 1 (2013).

10. W. T. Vestrand et al., Science 343, 38-41 (2014).

11. A. J. Levan, S. B. Cenko, D. A. Perley, N. R. Tanvir, GRB Coordinates Network 14455, 1 (2013).

12. R. Salvaterra et al., Astrophys. J. 749, 68 (2012).

13. S. E. Woosley, J. S. Bloom, Annu. Rev. Astron. Astrophys, 44, 507-556 (2006).

14. D. Xu et al., Astrophys. J. 776, 98 (2013).

15. M. Matsuoka et al., Publ. Astron. Soc. Jpn. 61, 999 (2009).

16. G. Tagliaferri et al., Nature 436, 985-988 (2005). 17. J. A. Nousek et al., Astrophys. J. 642, 389-400 (2006). 
18. P. Mészáros, Rep. Prog. Phys. 69, 2259-2321 (2006).

19. G. Ghisellini, M. Nardini, G. Ghirlanda, A. Celotti, Mon. Not. R. Astron. Soc. 393, 253-271 (2009)

20. A. Panaitescu, W. T. Vestrand, Mon. Not. R. Astron. Soc. 414, 3537-3546 (2011).

21. R. Sari, T. Piran, J. P. Halpern, Astrophys. J. 519, L17-L20 (1999).

22. H. van Eerten, W. Zhang, A. MacFadyen, Astrophys. J. 722, 235-247 (2010).

23. H. J. van Eerten, A. I. MacFadyen, Astrophys. J. 751, 155 (2012).

24. L. Nava, L. Sironi, G. Ghisellini, A. Celotti, G. Ghirlanda, Mon. Not. R. Astron. Soc. 433, 2107-2121 (2013)

25. H. van Eerten, A. van der Horst, A. MacFadyen, Astrophys. J. 749, 44 (2012).

26. R. Liu, X. Wang, X. Wu, Astrophys. J. Lett. 773, L20 (2013).

27. P.-H. T. Tam, Q.-W. Tang, S.-J. Hou, R.-Y. Liu, X.-Y. Wang, Astrophys. J. 771, L13 (2013).

28. L. Amati et al., Astron. Astrophys. 390, 81-89 (2002).

29. D. Yonetoku et al., Astrophys. J. 609, 935-951 (2004).
30. G. Ghirlanda, G. Ghisellini, D. Lazzati, Astrophys. J. 616, 331-338 (2004)

31. T. Laskar et al., Astrophys. J. 776, 119 (2013).

Acknowledgments: This work has been supported by ASI grant 1/004/11/0 and by Progetto di Ricerca di Interesse Nazionale (PRIN)-Ministero dell'Istruzione, dell'Università e della Ricerca (MIUR) grant 2009ERC3HT. Development of the BOXFIT code (25) was supported in part by NASA through grant NNX10AF62G issued through the Astrophysics Theory Program and by the NSF through grant AST-1009863. This research was partially supported by the Ministry of Education, Culture, Sports, Science and Technology of Japan (MEXT), grants-in-aid 14GS0211, 19047001, 19047003, and 24740186. The Liverpool Telescope is operated by Liverpool John Moores University at the Observatorio del Roque de los Muchachos of the Instituto de Astrofísica de Canarias. The Faulkes Telescopes, now owned by Las Cumbres Observatory are operated with support from the Dill Faulkes Educational Trust. Swift support at the University of Leicester and the
Mullard Space Science Laboratory is funded by the UK Space Agency. C.G.M. acknowledges financial support from the Royal Society, the Wolfson Foundation, and the Science and Technology Facilities Council. A.G. acknowledges funding from the Slovenian Research Agency and from the Centre of Excellence for Space Sciences and Technologies SPACE-SI, an operation partly financed by the European Union, European Regional Development Fund, and Republic of Slovenia. DARK is funded by the Danish National Research Foundation.

\section{Supplementary Materials}

www.sciencemag.org/content/343/6166/48/suppl/DC1

Materials and Methods

Supplementary Text

Figs. S1 to S7

Tables S1 to S10

References (32-62)

21 June 2013; accepted 16 October 2013

Published online 21 November 2013;

10.1126/science. 1242279

\section{The First Pulse of the Extremely Bright GRB 130427A: A Test Lab for Synchrotron Shocks}

R. Preece, ${ }^{1 *}{ }^{1 *}$ Michael Burgess, ${ }^{2 *}$ A. von Kienlin, ${ }^{3 *}$ P. N. Bhat, ${ }^{2}$ M. S. Briggs, ${ }^{2}$ D. Byrne, ${ }^{4}$ V. Chaplin, ${ }^{2}$ W. Cleveland, ${ }^{5}$ A. C. Collazzi, ${ }^{6,7}$ V. Connaughton, ${ }^{2}$ A. Diekmann, ${ }^{8}$ G. Fitzpatrick, ${ }^{4}$ S. Foley, ${ }^{4,3}$ M. Gibby, ${ }^{8}$ M. Giles, ${ }^{8}$ A. Goldstein, ${ }^{6,7}$ J. Greiner, $^{3}$ D. Gruber, ${ }^{3}$ P. Jenke, ${ }^{2}$ R. M. Kippen, ${ }^{9}$ C. Kouveliotou, ${ }^{6}$ S. McBreen, ${ }^{4,3}$ C. Meegan, ${ }^{2}$ W. S. Paciesas, ${ }^{5}$ V. Pelassa, ${ }^{2}$ D. Tierney, ${ }^{4}$ A. J. van der Horst, ${ }^{10}$ C. Wilson-Hodge, ${ }^{6}$ S. Xiong, ${ }^{2}$ G. Younes, ${ }^{5,6}$ H.-F. Yu, ${ }^{3}$ M. Ackermann, ${ }^{11}$ M. Ajello, ${ }^{12}$ M. Axelsson, ${ }^{13,14,15}$ L. Baldini, ${ }^{16}$ G. Barbiellini, ${ }^{17,18}$ M. G. Baring ${ }^{19}$ D. Bastieri, ${ }^{20,21}$ R. Bellazzini, ${ }^{22}$ E. Bissaldi, ${ }^{23}$ E. Bonamente, ${ }^{24,25}$ ]. Bregeon, ${ }^{22}$ M. Brigida, ${ }^{26,27}$ P. Bruel, ${ }^{28}$ R. Buehler, ${ }^{11}$ S. Buson, ${ }^{20,21}$ G. A. Caliandro, ${ }^{29}$ R. A. Cameron, ${ }^{30}$ P. A. Caraveo, ${ }^{31}$ C. Cecchi, ${ }^{24,25}$ E. Charles, ${ }^{30}$ A. Chekhtman, ${ }^{32}$ ]. Chiang, ${ }^{30}$ G. Chiaro, ${ }^{21}$ S. Ciprini, ${ }^{33,34}$ R. Claus, ${ }^{30}$ J. Cohen-Tanugi, ${ }^{35}$ L. R. Cominsky, ${ }^{36}$ ]. Conrad, ${ }^{37,14,38,39}$ F. D'Ammando, ${ }^{40}$ A. de Angelis, ${ }^{41}$ F. de Palma, ${ }^{26,27}$ C. D. Dermer, ${ }^{42 *}$ R. Desiante, ${ }^{17}$ S. W. Digel, ${ }^{30}$ L. Di Venere, ${ }^{30}$ P. S. Drell, ${ }^{30}$ A. Drlica-Wagner, ${ }^{30}$ C. Favuzzi, ${ }^{26,27}$ A. Franckowiak, ${ }^{30}$ Y. Fukazawa, ${ }^{43}$ P. Fusco, ${ }^{26,27}$ F. Gargano, ${ }^{27}$ N. Gehrels, ${ }^{44}$ S. Germani, ${ }^{24,25}$ N. Giglietto, ${ }^{26,27}$ F. Giordano, ${ }^{26,27}$ M. Giroletti, ${ }^{40}$ G. Godfrey, ${ }^{30}$ J. Granot, ${ }^{45}$ I. A. Grenier, ${ }^{46}$ S. Guiriec, ${ }^{44,7}$ D. Hadasch, ${ }^{29}$ Y. Hanabata, ${ }^{43}$ A. K. Harding, ${ }^{44}$ M. Hayashida, ${ }^{30,47}$ S. Iyyani, ${ }^{14,15,37}$ T. Jogler, ${ }^{30}$ G. Jóannesson, ${ }^{48}$ T. Kawano, $^{43}$ J. Knödlseder, ${ }^{49,50}$ D. Kocevski, ${ }^{30}$ M. Kuss, ${ }^{22}$ J. Lande, $^{30}{ }^{3}$. Larsson, ${ }^{15,14}$ S. Larsson, ${ }^{37,14,13}$ L. Latronico, $^{51}$ F. Longo, $^{17,18}$ F. Loparco, ${ }^{26,27}$ M. N. Lovellette, ${ }^{42}$ P. Lubrano, ${ }^{24,25}$ M. Mayer, ${ }^{11}$ M. N. Mazziotta, ${ }^{27}$ P. F. Michelson, ${ }^{30}$ T. Mizuno, ${ }^{52}$ M. E. Monzani, ${ }^{30}$ E. Moretti, ${ }^{15,14}$ A. Morselli, ${ }^{53}$ S. Murgia, ${ }^{30}$ R. Nemmen, ${ }^{44}$ E. Nuss, ${ }^{35}$ T. Nymark, ${ }^{15,14}$ M. Ohno, ${ }^{54}$ T. Ohsugi, ${ }^{52}$ A. Okumura, ${ }^{30,55}$ N. Omodei, ${ }^{30 *}$ M. Orienti, ${ }^{40}$ D. Paneque, ${ }^{56,30}$ ]. S. Perkins, ${ }^{44,57,58}$ M. Pesce-Rollins, ${ }^{22}$ F. Piron, ${ }^{35}$ G. Pivato, ${ }^{21}$ T. A. Porter, ${ }^{30}$ J. L. Racusin, ${ }^{44}$ S. Rainò, ${ }^{26,27}$ R. Rando, ${ }^{20,21}$ M. Razzano, ${ }^{22,59}$ S. Razzaque, $^{60}$ A. Reimer, ${ }^{23,30}$ O. Reimer, ${ }^{23,30}$ S. Ritz, ${ }^{59}$ M. Roth, ${ }^{61}$ F. Ryde, ${ }^{15}$ A. Sartori, ${ }^{31}$ J. D. Scargle, ${ }^{62}$ A. Schulz, ${ }^{11}$ C. Sgrò, ${ }^{22}$ E. J. Siskind, ${ }^{63}$ G. Spandre, ${ }^{22}$ P. Spinelli, ${ }^{26,27}$ D. J. Suson, ${ }^{64}$ H. Tajima, ${ }^{30,55}$ H. Takahashi, ${ }^{43}$ J. G. Thayer, ${ }^{30}$ J. B. Thayer, ${ }^{30}$ L. Tibaldo, $^{30}$ M. Tinivella, ${ }^{22}$ D. F. Torres, ${ }^{29,65}$ G. Tosti, ${ }^{24,25}$ E. Troja, ${ }^{44,66}$ T. L. Usher, ${ }^{30}$ ]. Vandenbroucke, ${ }^{30}$ V. Vasileiou, ${ }^{35}$ G. Vianello, ${ }^{30,67}$ V. Vitale, ${ }^{53,68}$ M. Werner, ${ }^{23}$ B. L. Winer, ${ }^{69}$ K. S. Wood, ${ }^{42}$ S. Zhu ${ }^{66}$

Gamma-ray burst (GRB) 130427A is one of the most energetic GRBs ever observed. The initial pulse up to 2.5 seconds is possibly the brightest well-isolated pulse observed to date. A fine time resolution spectral analysis shows power-law decays of the peak energy from the onset of the pulse, consistent with models of internal synchrotron shock pulses. However, a strongly correlated power-law behavior is observed between the luminosity and the spectral peak energy that is inconsistent with curvature effects arising in the relativistic outflow. It is difficult for any of the existing models to account for all of the observed spectral and temporal behaviors simultaneously.

I $n$ the context of Gamma-ray burst (GRB) 130427A, which triggered the Gamma-Ray Burst Monitor (GBM) (1) on the Fermi Gamma-
Ray Space Telescope on 27 April 2013 at $T_{0}=$ 07:47:06.42 UTC (2-4) is an extreme case. The peak flux on the 64-ms time scale is $1300 \pm$
100 photons $\mathrm{s}^{-1} \mathrm{~cm}^{-2}$ in the 10 to $1000 \mathrm{keV}$ range and the fluence, integrated over the same energy range and a total duration of $\sim 350 \mathrm{~s}$, is $(2.4 \pm$ $0.1) \times 10^{-3} \mathrm{erg} \mathrm{cm}^{-2}$. The longest continuously running GRB detector, Konus on the Wind spacecraft, has been observing the entire sky for nearly 18 years, and only one burst had a larger peak flux, by 30\% (GRB 110918A) (5). GRB 130427A is the most fluent burst in the era starting with the 1991 launch of the Burst and Transient Source Experiment (BATSE) on the Compton GammaRay Observatory. Finally, the energy of the spectral peak in the first time bin $\left(T_{0}-0.1\right.$ to $\left.0.0 \mathrm{~s}\right)$, $5400 \pm 1500 \mathrm{keV}$, is the second highest ever recorded (6).

The initial pulse (Fig. 1), lasting up to $2.5 \mathrm{~s}$ after the trigger, stands on its own as being so bright $\left(170 \pm 10\right.$ photons $\mathrm{s}^{-1} \mathrm{~cm}^{-2}$ peak flux for 10 to $1000 \mathrm{keV}$ in the $64-\mathrm{ms}$ time bin at $T_{0}+0.51 \mathrm{~s}$ ) as to be ranked among the 10 brightest GBM or BATSE bursts (7-9). The brightness allows us to track the spectral evolution of the rising portion of a well-separated pulse with unprecedented detail (10). Evident in the GBM low-energy light curve [Fig. 1; as well as the 15 to $350 \mathrm{keV}$ light curve presented in (11)] are fluctuations starting at around $1 \mathrm{~s}$ that are not present at higher energies. If these represent additional low-energy pulses, their presence clearly does not dominate the analyses presented below.

Past studies of time-resolved spectra of simple pulses in GRBs indicate that there are broadly two classes of spectral evolution. These are called "hard-to-soft" and "tracking" pulses $(12,13)$, depending on whether the energy of the peak in the $v F_{v}$ spectrum (generically called $E_{\text {peak }}$ herein) monotonically decays independently of the flux evolution or else generally follows the rise and fall of the flux. Typically, there are at most one or two spectra available for fitting during the rising portion of the flux history. What makes this event unique is that there are roughly six time bins with excellent counts statistics before the peak in the 10 to $1000 \mathrm{keV}$ flux.

As seen in Fig. 1, there is a clear trend in the individual detector's light curves: the $>20 \mathrm{MeV}$ 
GRB 130427A: A Nearby Ordinary Monster

A. Maselli et al.

Science 343, 48 (2014);

DOI: $10.1126 /$ science. 1242279

This copy is for your personal, non-commercial use only.

If you wish to distribute this article to others, you can order high-quality copies for your colleagues, clients, or customers by clicking here.

Permission to republish or repurpose articles or portions of articles can be obtained by following the guidelines here.

The following resources related to this article are available online at www.sciencemag.org (this information is current as of May 8, 2015 ):

A correction has been published for this article at:

http://www.sciencemag.org/content/343/6174/969.2.full.html

Updated information and services, including high-resolution figures, can be found in the online version of this article at:

http://www.sciencemag.org/content/343/6166/48.full.html

Supporting Online Material can be found at:

http://www.sciencemag.org/content/suppl/2013/11/20/science.1242279.DC1.html

A list of selected additional articles on the Science Web sites related to this article can be found at:

http://www.sciencemag.org/content/343/6166/48.full.html\#related

This article cites $\mathbf{5 5}$ articles, 17 of which can be accessed free:

http://www.sciencemag.org/content/343/6166/48.full.html\#ref-list-1

This article has been cited by 7 articles hosted by HighWire Press; see:

http://www.sciencemag.org/content/343/6166/48.full.html\#related-urls

This article appears in the following subject collections:

Astronomy

http://www.sciencemag.org/cgi/collection/astronomy 